\title{
EBSP, PROGRESS IN TECHNIQUE AND APPLICATIONS
}

\author{
J. HJELEN, $\dagger$ R. ØRSUND, $\ddagger$ E. HOEL, P. RUNDE,§ T. FURU§ \\ and E. NES" \\ †Royal Norwegian Air Force Academy, N-7004 Trondheim mil, Norway \\ $\ddagger$ Hydro Aluminium $A / S, N$-6601 Sunndals $\phi r a$, Norway \\ $\S$ SINTEF, Division of Metallurgy, N-7034 Trondheim, Norway \\ /I The Norwegian Institute of Technology, Department of Metallurgy, N-7034 \\ Trondheim, Norway
}

\begin{abstract}
To measure grain orientation in SEM with high accuracy a new EBSP calibration software is developed. Grain orientation determination is simply based on marking three zone axes in the live EBSP. The EBSP-technique has been applied to study misorientation between subgrains in high purity and commercially pure aluminium. The lattice curvatures, which develop during deformation, increase with the amount of deformation. During annealing the subgrains grow, resulting in an increased average neighbouring misorientation due to misorientation accumulation.
\end{abstract}

KEY WORDS EBSP, computer program, orientation determination, aluminium, subgrain misorientation, recovery.

\section{INTRODUCTION}

Textures of crystalline materials are traditionally determined from bulk specimens using X-ray diffraction. These textures are macrotextures from large volumes. However, the relationship between the texture and the local microstructure is required to understand the development of textures. The analysis has then to be carried out in electron microscopes. The problems associated with the determination of microtextures using electron beam techniques have been described by Humphreys (1988).

This paper describes recent developments in electron backscattering patterns (EBSPs) software and applications of the EBSP-technique to study the deformation structure and processes occurring during the very early period of annealing of heavily deformed structures. This involves a detailed study of the changes in the substructure with annealing time. This is now possible due to the EBSP-technique in SEM in combination with the convergent beam technique in TEM, which provide a very powerful method of obtaining structural information on a microscale. The investigation was carried out on heavily deformed and slightly annealed aluminium, both of high purity and commercial purity quality. In this study of the recovery process, the extent of recovery is determined by means of the changes in subgrain size and subboundary misorientation with annealing time. 


\section{EBSP-SOFTWARE}

When using EBSP in texture analyses it is important to have effective software which provides fast and accurate orientation determination and data collection. We here describe a software system for semi-automatic on-line analysis of EBSP's for cubic materials.

\section{CALIBRATION PROCEDURE}

Most of the parameters in the calibration data can be calculated from the hardware system or can be read directly from the SEM control panel. This applies, for instance, to the camera magnification, working distance and specimen tilt angle. However, two important parameters must be accurately determined by a calibration procedure. They are the beam normal position on the phosphor screen and the distance between the specimen and the screen. The most common method for the determination of these two parameters involves the use of a large single crystal, usually silicon, see Dingley et al. (1987). The main disadvantage is that it is necessary to know the precise orientation of this crystal in the SEM during calibration. This unavoidable inaccuracy introduces an error in the beam normal position which later results in errors in absolute orientation measurements.

Figure 1 illustrates an alternative method, Hjelen et al. (1991a), for determination of the beam normal position, BN. The beam normal is defined as being normal to the phosphor screen as well as to the incoming electron beam. It will therefore intersect the screen at a position independent of the specimen to screen distance. The first step in the calibration procedure is to pull the phosphor screen

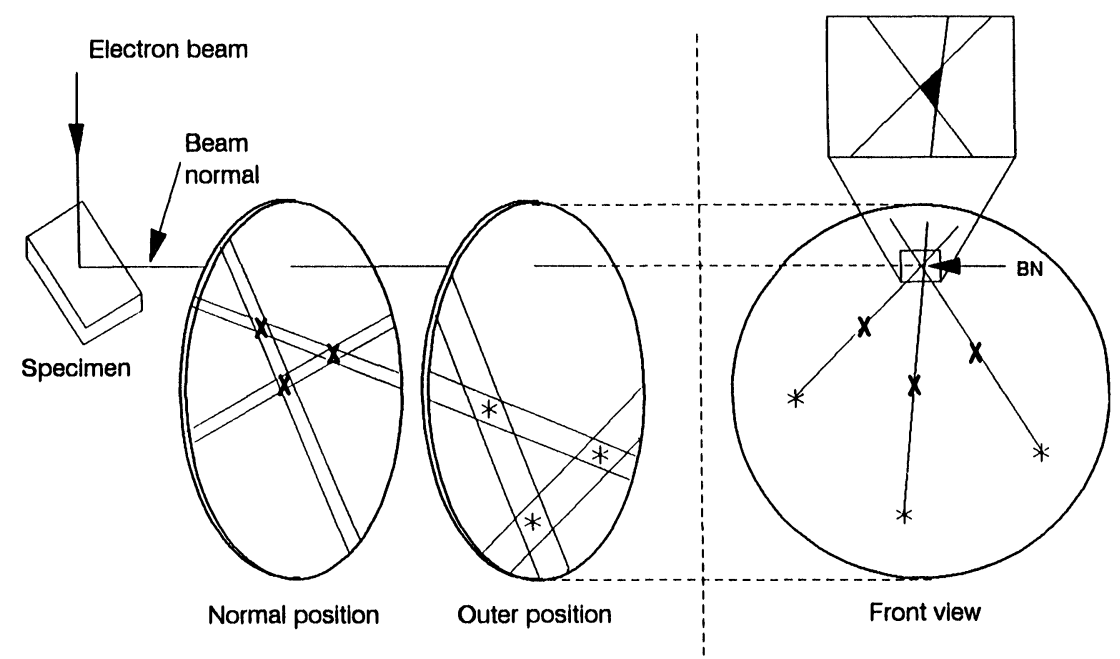

Figure 1 Three zone axes are marked with the phosphor screen in two different positions. The three straight lines seen in the front view of the screen will forma a triangle used to calculate the beam normal position, $\mathrm{BN}$, and the accuracy of the result. 


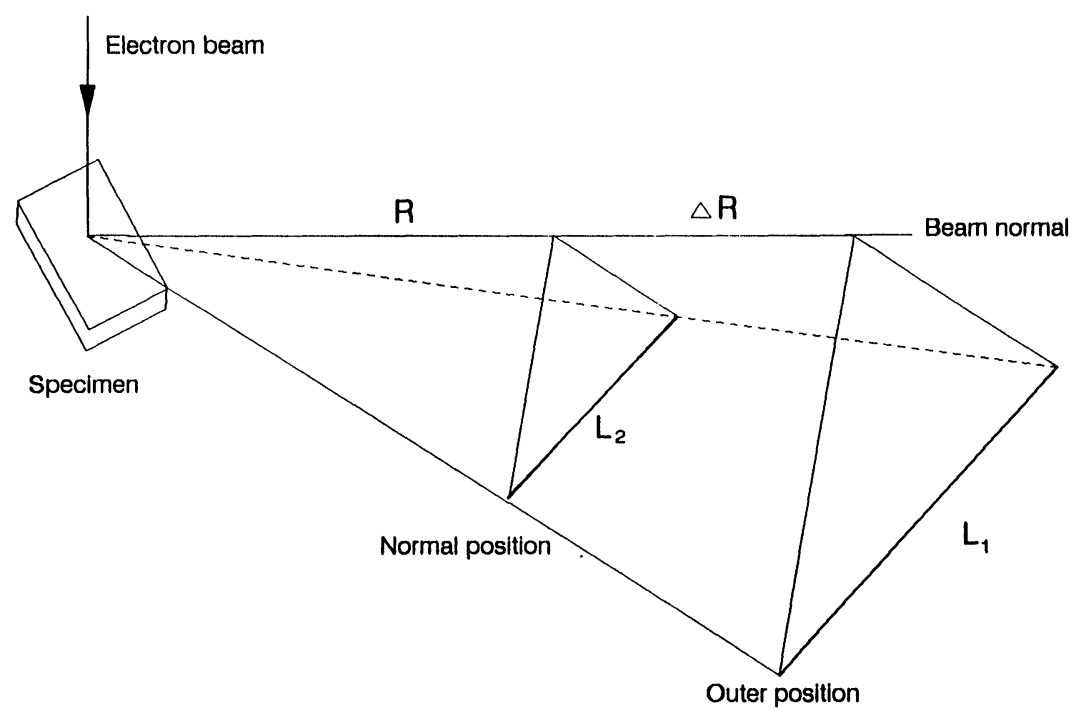

Figure 2 Distances used to calculate $R$ in Eq. (1). Only two of the three zone axes in Figure 1 are included.

to a predefined outer position and locate three zone axes in the diffraction pattern. The next step is to put the screen back to its normal operating position and the locations of the same three zone axes are determined once more. The two points of each zone axis can then be used to form a straight line, and three such lines intersect at the beam normal position. This is shown in the front view of the screen in Figure 1. It is, of course, only necessary to have two such lines to determine $\mathrm{BN}$, but the use of three or more lines reduces any errors and makes it possible to estimate the accuracy of the results. Furthermore, it is not necessary to use zone axes for the determination, any easily recognizable points in the pattern will do. The same points in the pattern can also be used to determine the distance $R$ between the specimen and the screen, Figure 2. After calculating the distances $L_{1}$ and $L_{2}$ between two zone axes, $R$ can be found from the relation

$$
\frac{L_{2}}{R}=\frac{L_{1}}{R+\Delta R}
$$

where $\Delta R$ is the distance from the outer to the normal screen position. Two additional estimates for $R$ can be found if the third zone axis is introduced in the computations.

This calibration procedure deviates from earlier available methods in the sense that it can be carried out with any specimen and is independent of its orientation. The operator does not need any knowledge of electron diffraction maps and the calibration is only necessary the first time the system is set up.

\section{DATA ACQUISITION}

To calculate the crystallographic orientation of a grain, the operator simply identifies the positions of three strong zone axes in the diffraction pattern, Figure 


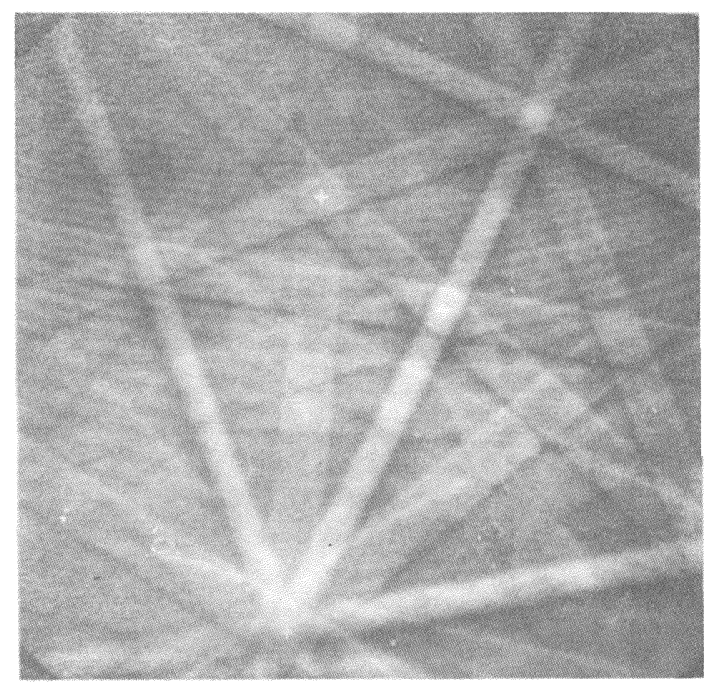

Figure 3 EBSP of aluminium, three zone axes are marked for orientation determination.

3. This is done by superimposing a mouse-controlled icon on the live EBSPimage. The program then calculates the three interzone angles. The next step is to search in an appropriate database (FCC or BCC) to find the indices of the three zones. When the best fit between the experimental angles and the exact angles in the database is found, the crystallographic orientation is calculated. The position and indices of other zone axes present in the EBSP are superimposed on the monitor, Figure 4, and the operator is asked to accept or reject the proposed indexing. If accepted, the operator can move the electron beam to the next grain

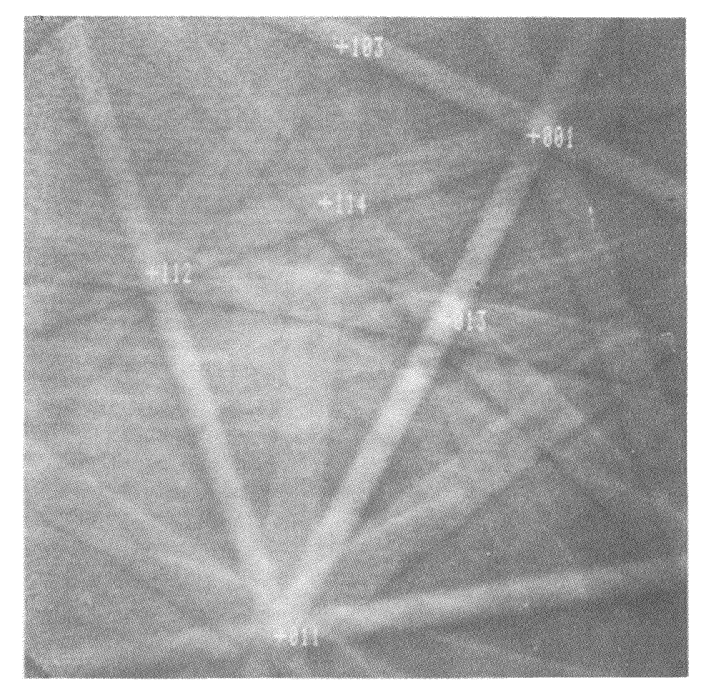

Figure 4 Simulated pattern superimposed on the EBSP. 
and start analysing the next EBSP. If the proposed indexing is not accepted, the second best fit will come up. A $3 \times 3$ matrix describing the absolute crystallographic orientation of the grain as described by Bunge (1982) is shown together with the corresponding pole figure. The misorientation between the present grain and the previous grain is also presented on the computer monitor for every grain.

The time taken to determine the orientation of a grain is less than one second using an IBM-compatible 80486 PC. In addition it takes about 15 seconds to mark the zone axes. The indexing first proposed is correct in approximately $95 \%$ of the cases, but if no correct indexing can be found, it is possible to change the database and start a new indexing sequence. This might be useful in materials containing both FCC and BCC grains, for instance duplex steel.

\section{ACCURACY}

A displacement in beam normal position leads to an absolute error in orientation determinations. With the calibration routine described above, the beam normal position was measured with an uncertainty of $0.7 \mathrm{~mm}$ for a specimen to screen distance of $30 \mathrm{~mm}$. This resulted in $1.3^{\circ}$ uncertainty in the absolute orientation. Error due to non-linearity in the camera and uncertainty in the screen angle were estimated to be less than $1^{\circ}$. Although the mounting of the specimen used for calibration does not introduce any errors in absolute orientation, a shift in the working distance when using a different specimen for orientation determination will lead to such errors. This can be corrected if the precise working distance is known. However, the working distance displayed on the SEM control panel is not exact and can introduce an additional absolute error of about $1^{\circ}$. This error can be eliminated in two ways, the easiest of which is to use the same objective lens current as was used during calibration, and instead focus by adjusting the specimen height. If a different working distance is required, a new calibration can simply be carried out without changing the specimen. In addition to the errors already mentioned, uncertainty in specimen mounting and tilt angle will introduce absolute errors in the orientation. When all these sources of errors are considered, uncertainty of absolute orientation is estimated to be about $2^{\circ}-4^{\circ}$.

Relative orientation measurements are often of more use than absolute orientation. The error involved is smaller and can be found by indexing the same pattern several times and calculating the misorientation. In the system described here, the uncertainty for relative orientation measurements is estimated to be $0.4^{\circ}$.

\section{LOCAL LATTICE CURVATURES}

It is well established that heavily cold worked metals consist of cells or subgrains which are slightly misoriented with respect to each other. During annealing, recovery takes place, both before and simultaneously with recrystallization. During the recovery process, the substructure will experience a general coarsening, due to subgrain growth. 


\section{EXPERIMENTAL TECHNIQUES}

In this investigation, two commercial AA1100-series aluminium alloys with their chemical compositions given in Table 1 have been studied. In addition, high purity aluminium polycrystals were included in this investigation. Regarding the high purity aluminium polycrystals, this material was produced by directional solidification, resulting in large columnar crystals in the $\mathrm{mm}$ range. The CPA1 alloy and the high purity aluminium were cold rolled in a laboratory rolling mill to $90 \%$ reduction, and the subsequent heat treatments were carried out in salt baths followed by water quenching, ensuring a rapid heating- and cooling rate of the material. Regarding the CPA2 alloy, the cold rolling sequence was carried out in a full scale plant. Specimens for microtexture investigations were taken from the transverse section defined by the rolling direction and the sheet plane normal.

The EBSP-equipment was attached to a Jeol 840 SEM and the stationary beam was digitally moved both parallel and perpendicular to the rolling direction. For further details about the EBSP-technique, see Hjelen (1992). The SEM settings were selected to ensure the best possible resolution of the EBSP-equipment, see Hjelen and Nes (1990), and the specimens were rotated around the TD-direction. This was carried out due to the elongation of the subgrains in the rolling direction, where the resolution does not have to be as good as in the normal direction of the specimen. In order to obtain detailed information about the orientation changes from subgrain to subgrain, the electron beam was moved in steps of approximately $0.1 \mu \mathrm{m}$ to ensure that every subgrain along a line was taken into account. In order to quantify the accumulation of misorientation built up during deformation, two techniques have been applied, which may be explained as follows:

i) Line scans, see Ørsund et al. (1989). Figure 5 (left) shows a schematic presentation of a rolled plate with planes and directions defined. The specimens for microtexture investigation were taken from the transverse section defined by the rolling direction (RD) and the sheet plane normal (ND). Figure 5 (right) shows a magnified view of the substructure developed in this section, with pancake shaped subgrain elongated in the rolling direction. The procedure here is to scan both perpendicular and parallel to the rolling direction and collect the orientation of each subgrain scanned. The tendency to accumulation of misorientation has been quantified by measuring the orientation of the subgrains and calculate the misorientation angle between a reference subgrain and the other subgrains. The misorientations are plotted versus distance as shown in Figure 6.

Table 1 Chemical composition of the commercial pure aluminium.

\begin{tabular}{lllll}
\hline Alloy & $\begin{array}{l}\text { Casting } \\
\text { technique }\end{array}$ & \multicolumn{3}{c}{ Alloy composition [wt\%] } \\
\cline { 3 - 5 } & & $F e$ & $S i$ & $A l$ \\
\hline CPA1 & DC & 0.29 & 0.12 & Bal. \\
CPA2 & DC & 0.43 & 0.09 & Bal. \\
\hline
\end{tabular}



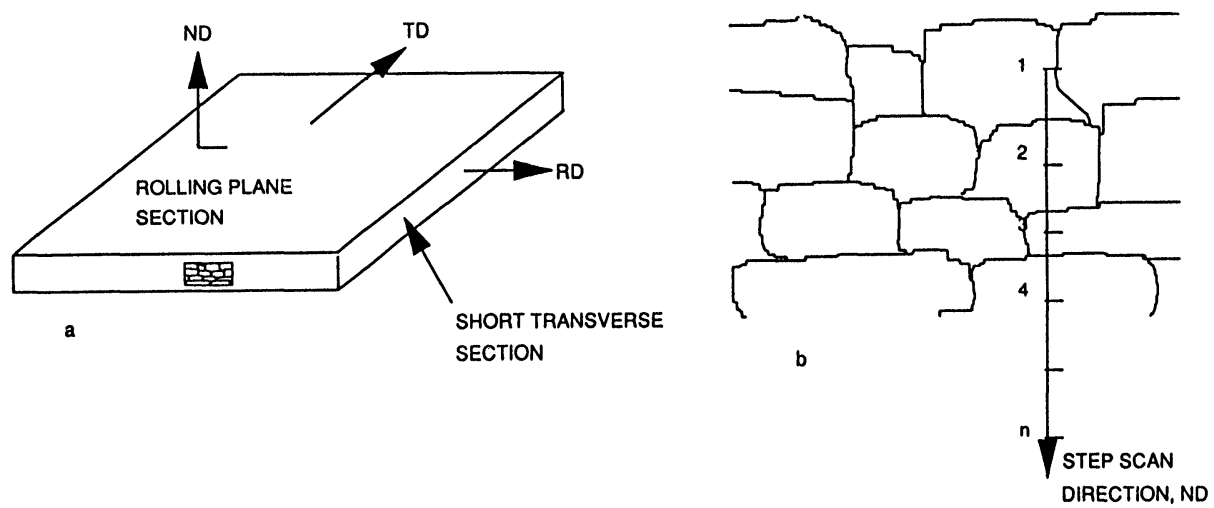

Figure 5 Schematic presentation of a rolled plate (a) with a magnified view of the substructure (b).

ii) Misorientation vs. subgrain size. This technique is a direct measurement of the average subgrain misorientation, i.e. quantification of how the parameter changes with subgrain size during annealing. This technique has been applied on the commercial purity metals.

\section{EXPERIMENTAL RESULTS AND DISCUSSION}

The results of subgrain size and subboundary misorientation vs. annealing time, and subboundary misorientation vs. subgrain size for the commercial purity alloys are given in Figure 7 . In the case of the CPA2 alloy, the annealing has been carried out at $300^{\circ} \mathrm{C}$, while the annealing temperature was $325^{\circ} \mathrm{C}$ for the CPA1 alloy. Regarding the high purity aluminium, the results for both single crystals and polycrystals, deformed to true strains of 1 and 2.3 respectively, are given in Figure 8. The results for the single crystals, after channel die compression, are
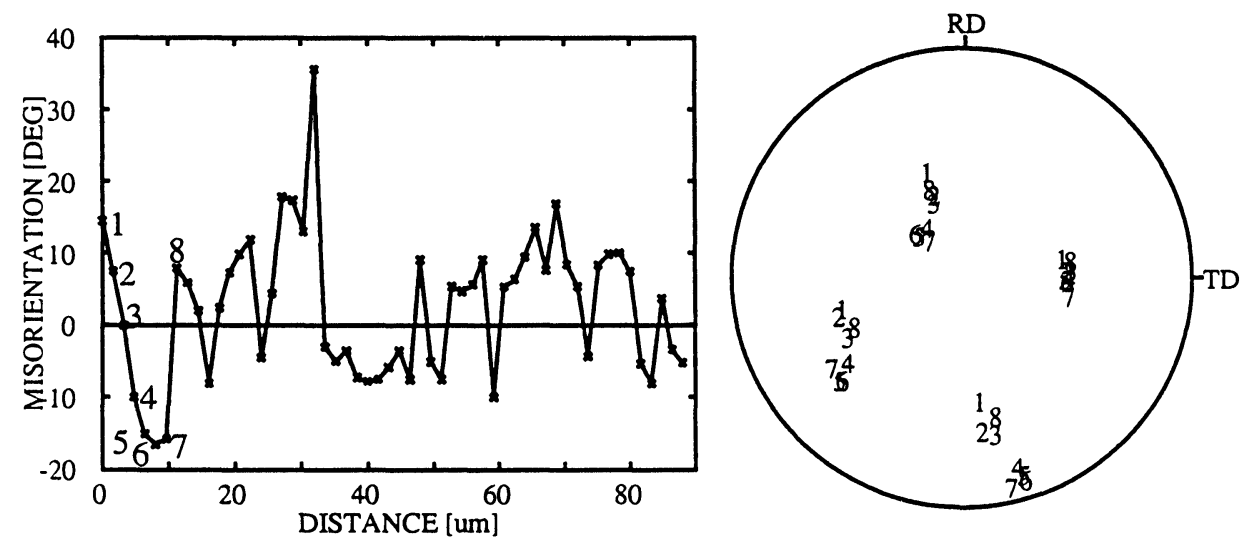

Figure 6 Lattice rotation as a function of distance. 

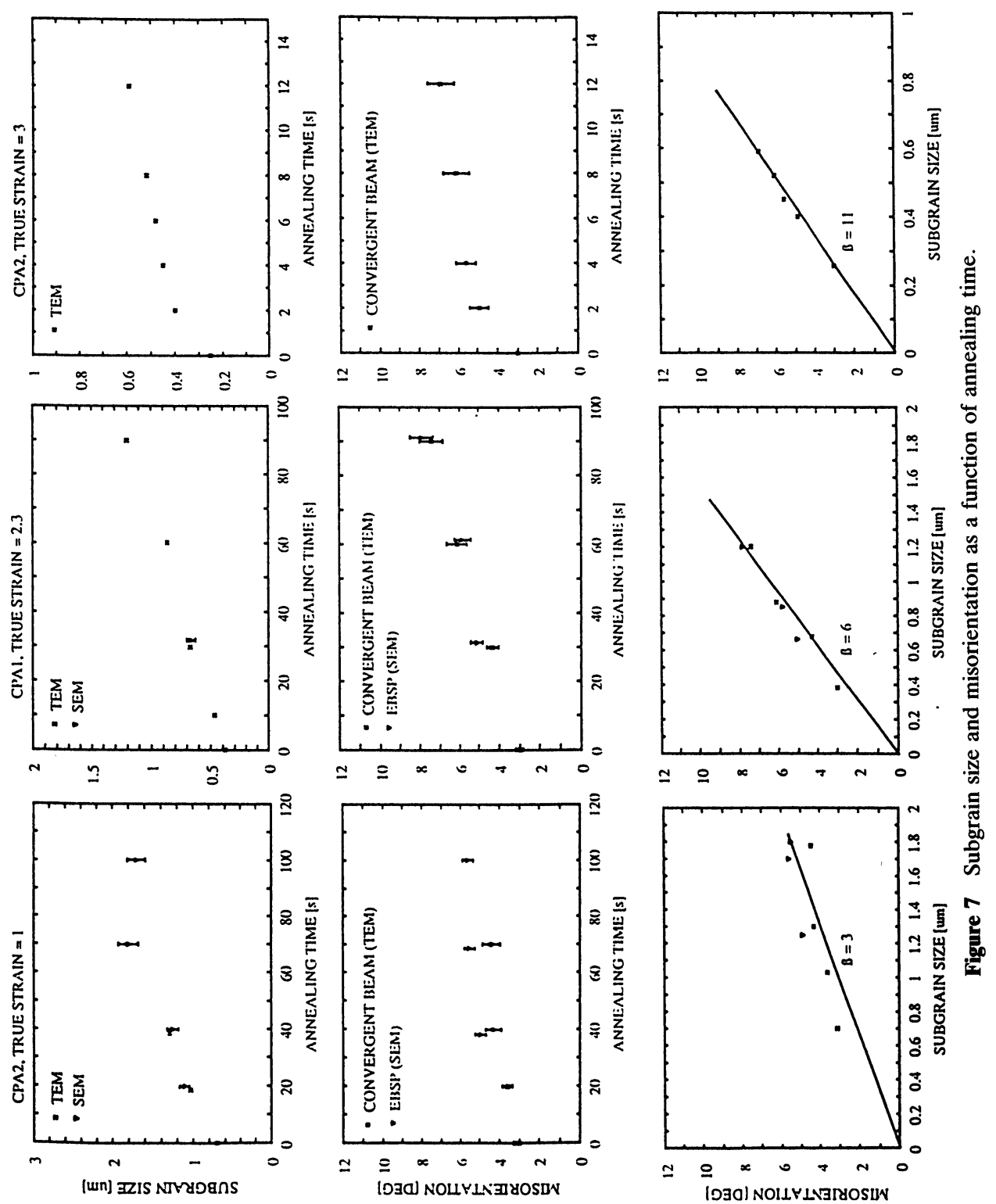

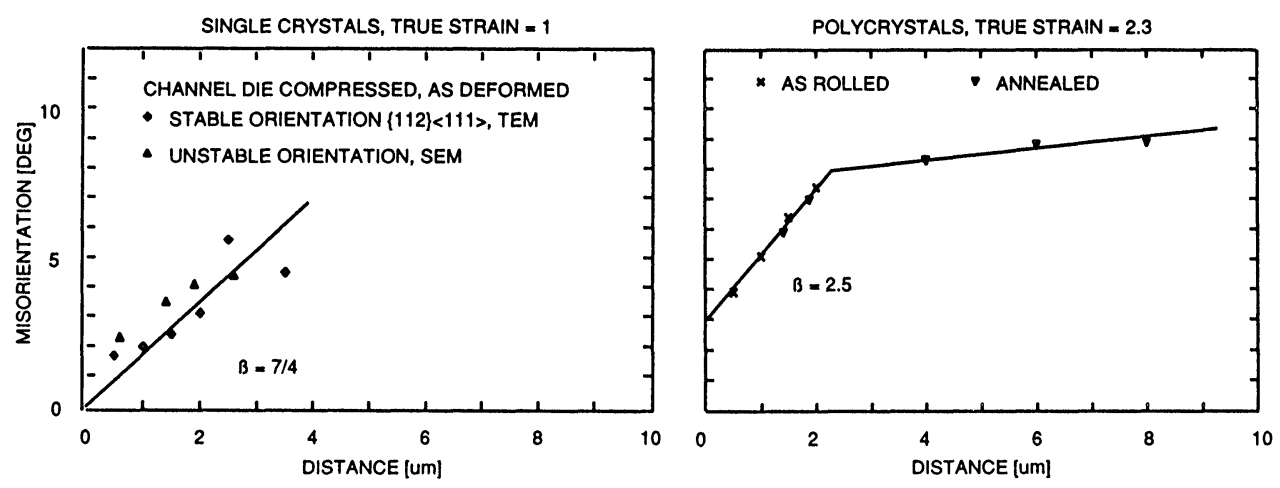

Figure 8 Misorientation gradient in single crystals (left) and polycrystals (right), high purity aluminium. Step scan direction || ND.

taken from an investigation by Hjelen et al. (1991c). The annealing treatment of the high purity aluminium polycrystals was carried out at $400^{\circ} \mathrm{C}$.

As can be seen from the diagrams presented in Figure 7, where the subboundary misorientation is given as a function of annealing time, the average subboundary misorientation between subgrains seems to increase with increased annealing time. These results indicate that the misorientation between neighbouring subgrains will increase when the subgrain structure gets coarser, indicating that a lattice curvature of some extent and range is built up during deformation and is accumulated in the subgrain boundaries during the growth process. The results presented in Figure 7 show the changes in both the size of the subgrains and the misorientation between subgrains during post deformation annealing. In Figure 8 however, where the results for high purity aluminium are presented, the polycrystalline material has been investigated both in the as deformed condition and after a minor annealing treatment. It should also be mentioned that the results presented in Figure 8 are not neighbouring misorientations alone, but also show the misorientation up to the fifth nearest neighbour. In the case of the polycrystalline material, investigated by the EBSP-technique, it may be seen that the results for the annealed material directly fit into the measurements of the material in the as deformed condition. This observation provides clear evidence for the development of a lattice curvature during deformation, because if the rotation was of a statistical nature, the results from investigations on annealed material should not be expected to fit into the results for the material in the as deformed condition. Then the rotation would have to be caused by statistically stored dislocations whose density would have been affected by the annealing, and the lattice curvature should more or less disappear during the recovery process. The results indicate that this is not the case, and consequently the lattice rotation is caused by geometrically stored dislocations, which are not as sensitive to annealing treatments, resulting in a maintenance of the curvature developed during deformation. When a subgrain experiences a coarsening due to subgrain growth, it will therefore have to accumulate this rotation, resulting in an increased boundary misorientation. Another important aspect that has to be mentioned regarding the results for the HPA polycrystals in Figure 8 is that the nearest neighbour misorientation seems to saturate at a value 
close to $9^{\circ}$, which is about three times the misorientation in the as rolled condition. The same tendency was also observed by Daaland (1992), and this saturation value is expected to depend on both the amount of cold reduction and the initial grain size of the material.

An alternative way of presenting the results of misorientation accumulation is shown in Figure 9. The results are from a high purity aluminium single crystal deformed to a true strain of 1.0 and examined in the as rolled condition. By systematically step scanning the electron beam (step lengths less than $0.5 \mu \mathrm{m}$ ), the local variation in orientation over relatively large distances can be mapped with a high degree of accuracy. The result of one such scan is presented in terms of a "continuous-orientation-change-curve" and a (111)-pole figure in Figure 9. This "zig-zag" curve represents a recording of the orientation changes from subgrain to subgrain, with reference to a mean orientation. As the mean orientation we have selected a grain at the centre of the orientation distribution of all subgrains (i.e. the centre of the orientation spread of the local texture component, in this case close to the $\mathrm{Cu}$-component). Further, the angular deviation of a specific subgrain (which is recorded on the vertical axis in Figure 10) represents the angle of the rotation around a non-specified axis which this subgrain in question will have to be rotated to coincide with the reference orientation. With respect to the reference orientation the curve in Figure 9 simply represents a misorientation-plot. Regarding the orientation of the non-specified axis of orientation, it follows from the EBSP-examination that we have two main axes around which the subgrains are scattered in an alternating way. These axes are either of the $\langle 111\rangle$-axis or the TD-axis. This rotation-pattern can easily be seen from the EBSP-pole figure on the right hand side in Figure 9. It follows from the misorientation plot that the orientation spread is not statistical around a mean orientation in a random way. It is rather in the form of local accumulation of frequently amount to deviations from the mean of $20^{\circ}-30^{\circ}$ or more. For more details, see Hjelen et al. (1991b).

The results for all the materials and variants investigated are condensed into the diagram presented in Figure 10, where the boundary misorientation is plotted
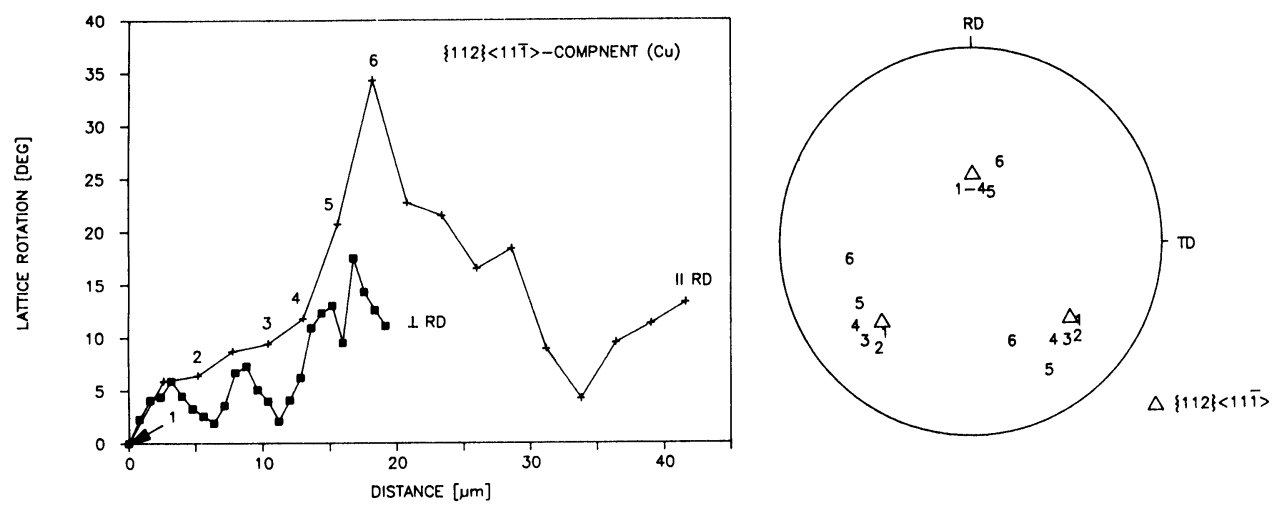

Figure 9 Lattice rotation measured from step scan in high purity aluminium. Step scan direction \| ND. 


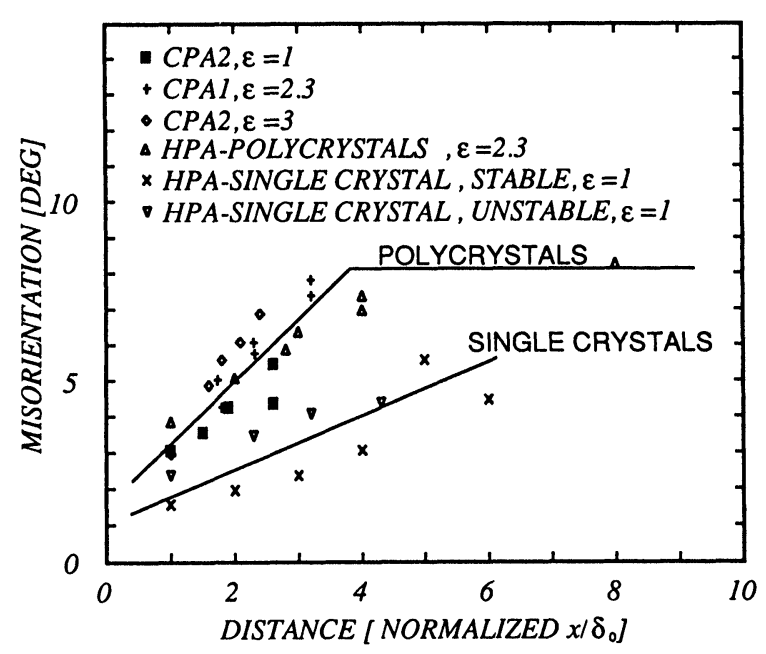

Figure 10 Misorientation as a function of distance for single- and polycrystals, commercial and high purity aluminium.

as a function of the normalized subgrain size, where " $\delta_{0}$ " is the average subgrain size in the as rolled condition. When plotted in such a way, it turns out that the materials may be sorted as belonging to two different categories, namely as polyand single crystalline materials. A single crystalline material seems to obtain a smaller lattice rotation compared to polycrystalline materials, even if the same amount of deformation reduction has been applied.

\section{CONCLUSIONS}

-EBSP calibration software that can be used with any polycrystalline specimen is developed.

- Grain orientation determination is based on marking three zone axis in the live EBSP.

-During deformation, a localized lattice curvature develops, the extent of which seems to increase with amount of deformation (increased value of $\beta$ ).

- The rotational gradients seem to be more pronounced in the polycrystals compared with single crystalline material, even at the same amount of deformation.

-During subgrain growth, the experimental results indicate that the lattice curvature during deformation is preserved, resulting in an increased average neighbouring misorientation due to misorientation accumulation.

\section{References}

Bunge, H. J. (1982). Texture Analysis in Material Science, Butterworths \& Co.

Daaland, O. (1992). To be published. 
Dingley, D. J., Longden, M., Weinbren, J. and Alderman, J. (1987). On-line analysis of electron backscatter diffraction patterns. I. Texture analysis of zone refined polysilicon. Scanning Microscopy. 1, 2, 451-456.

Hjelen, J. (1992). EBSP-A powerful SEM-technique to study local textures in metallic materials. To be published.

Hjelen, J. and Nes, E. (1990). Spatial resolution measurements of electron backscatter diffraction patterns (EBSPs) in the scanning electron microscope. Proc. of the XII Int. Cong. for Electron Microscopy, San Francisco Press, Inc., pp. 404-405.

Hjelen, J., Hoel, E. and Ørsund, R. (1991a). Electron diffraction in the SEM. Micron and Microscopia Acta, 22, 1/2, 137-138.

Hjelen, J., Ørsund, R. and Nes, E. (1991b). On the origin of recrystallization textures in aluminium. Acta Metall. Mater. 39, 7, 1377-1404.

Hjelen, J., Weiland, H., Butler, J., Liu, J., Hu, H. and Nes, E. (1991c). The orientation distribution in channel die compressed Al single crystals as studied by microdiffraction in SEM and TEM. Textures and Microstructures. 14-18, 983-988.

Humphreys, F. J. (1988). Experimental techniques for microtexture determination. Proc. Eight Int. Conf. on Textures of Materials, (ICOTOM 8), Kallend, J. S. and Gottstein, G. Eds., The Metallurgical Society. Warrendale Pa. pp. 171-182.

Ørsund, R., Hjelen, J. and Nes, E. (1989). Local lattice curvature and deformation heterogeneities in heavily deformed aluminium. Scripta Met., 23, 1193-1197. 